\title{
Phase structure of a generalized Nambu-Jona-Lasinio model with Wilson fermions in the mean-field or large- $N$ expansion
}

\author{
V. Azcoiti ${ }^{a}$, G. Di Carlo ${ }^{b}$, E. Follana ${ }^{a}$, M. Giordano ${ }^{c}$, A. Vaquero ${ }^{\mathrm{d}, *}$ \\ a Departamento de Física Teórica, Facultad de Ciencias, Universidad de Zaragoza, Cl. Pedro Cerbuna 12, \\ E-50009 Zaragoza, Spain \\ b INFN, Laboratori Nazionali del Gran Sasso, I-67100 Assergi, L'Aquila, Italy \\ ${ }^{\mathrm{c}}$ Institute of Nuclear Research of the Hungarian Academy of Sciences (ATOMKI), Bemtér 18/c, \\ H-4026 Debrecen, Hungary \\ d Computation-based Science and Technology Research Center (CaSToRC), The Cyprus Institute, \\ 20 Constantinou Kavafi Street, Nicosia 2121, Cyprus
}

Received 10 April 2013; accepted 1 July 2013

Available online 9 July 2013

\begin{abstract}
We analyze the vacuum structure of a generalized lattice Nambu-Jona-Lasinio model with two flavors of Wilson fermions, such that its continuum action is the most general four-fermion action with "trivial" color interactions, and having a $S U(2)_{V} \times S U(2)_{A}$ symmetry in the chiral limit. The phase structure of this model in the space of the two four-fermion couplings shows, in addition to the standard Aoki phases, new phases with $\left\langle\bar{\psi} \gamma_{5} \psi\right\rangle \neq 0$, in close analogy to similar results recently suggested by some of us for lattice QCD with two degenerate Wilson fermions. This result shows how the phase structure of an effective model for low-energy QCD cannot be entirely understood from Wilson Chiral Perturbation Theory, based on the standard QCD chiral effective Lagrangian approach.
\end{abstract}

(C) 2013 Elsevier B.V. All rights reserved.

Keywords: Nambu-Jona-Lasinio; Lattice QCD; Wilson fermions; Spontaneous symmetry breaking; Parity; Flavor symmetry

\footnotetext{
* Corresponding author.

E-mail address: a.vaquero@cyi.ac.cy (A. Vaquero).
} 


\section{Introduction}

Since the first numerical investigations of four-dimensional non-abelian gauge theories with dynamical Wilson fermions were performed in the early 80 s $[1,2]$, the understanding of the phase and vacuum structure of lattice QCD with Wilson fermions at nonzero lattice spacing, and of the way in which chiral symmetry is recovered in the continuum limit, has been a goal of lattice field theorists. The complexity of the phase structure of this model has been known for a long time. The existence of a phase with parity and flavor symmetry breaking was conjectured for this model by Aoki in the middle 80s [3,4], and confirmed later on [5-27].

The standard wisdom on lattice QCD with Wilson fermions is that even if chiral symmetry is explicitly broken at finite lattice spacing $a$ by the Wilson regularization, this symmetry will be recovered and spontaneously broken in the continuum limit. However, it is difficult to understand why there exists a critical line at finite lattice spacing along which the three pions are massless. Indeed, the pions cannot be the three Goldstone bosons associated with the spontaneous breaking of the $S U(2)$ chiral symmetry since, as previously stated, the Wilson regularization breaks explicitly this symmetry.

One of the main features of Aoki's picture was to clarify this point. In the Aoki phase, the charged pions are massless because they are the two Goldstone bosons associated with the spontaneous breaking of the $S U(2)$ flavor symmetry down to $U(1)$, with a non-vanishing vacuum expectation value of the $i \bar{\psi} \gamma_{5} \tau_{3} \psi$ condensate. The neutral pion, which is massive in the Aoki phase, becomes massless on the critical line because flavor symmetry is continuously recovered on this line which separates the broken (Aoki) phase from the unbroken (physical) phase. The other relevant feature of the Aoki scenario is that it provides a counterexample to the Vafa-Witten theorem on the impossibility to spontaneously break parity in a vector-like theory with positive definite integration measure [28-32].

Aoki's conjecture has been supported not only by numerical simulations of lattice QCD, but also by theoretical studies based on the Nambu-Jona-Lasinio model [5,33], on the linear sigma model [6], and on applying Wilson Chiral Perturbation Theory $(W \chi P T)$ to the continuum effective Lagrangian [11]. The latter analysis predicts, near the continuum limit, two possible scenarios, depending on the sign of an unknown low-energy coefficient. In the first scenario, flavor and parity are spontaneously broken, and there is an Aoki phase with a nonzero value only for the $i \bar{\psi} \gamma_{5} \tau_{3} \psi$ condensate, whereas $\left\langle i \bar{\psi} \gamma_{5} \psi\right\rangle=0$. In the other one (the "first-order" scenario) there is no spontaneous symmetry breaking.

This standard picture for the Aoki phase was questioned by three of us in [21], where we conjectured on the appearance of new vacua in the Aoki phase, which can be characterized by a non-vanishing vacuum expectation value of the flavor-singlet pseudoscalar condensate $i \bar{\psi} \gamma_{5} \psi$, and which cannot be connected to the Aoki vacua by parity-flavor symmetry transformations. More recently, we have obtained results from numerical simulations of lattice QCD with two degenerate flavors of Wilson fermions, suggesting that our conjecture could be realized [34]. Since these results seem to question the validity of the $W \chi P T$ analysis [11], an approach which has been successfully applied in many contexts, it is worthwhile to analyze the possible origins of this discrepancy.

First, one should notice that the chiral effective Lagrangian approach is based on the continuum effective Lagrangian written as a series of contributions proportional to powers of the lattice spacing $a$, plus the construction of the corresponding chiral effective Lagrangian, keeping only the terms up to order $a^{2}$ [11]. This means that predictions based on this chiral effective Lagrangian approach should work close enough to the continuum limit, where keeping terms 
only up to order $a^{2}$ can be justified. However, the data reported in [34] were obtained at $\beta=2.0$, and a very rough estimate gives a lattice spacing of order $3.0 \mathrm{GeV}^{-1}$ at this $\beta$. Hence, a possible explanation for the discrepancies that we found relies on the necessity of including higher-order terms in the chiral effective Lagrangian.

We want to recall here that the Aoki effective potential was obtained from a strong coupling expansion combined with a $1 / N$ expansion [4], i.e., far away from the continuum limit. Furthermore, Aoki's solution shows degenerate vacua with $\left\langle i \bar{\psi} \gamma_{5} \tau_{3} \psi\right\rangle \neq 0,\left\langle i \bar{\psi} \gamma_{5} \psi\right\rangle=0$ and $\left\langle i \bar{\psi} \gamma_{5} \tau_{3} \psi\right\rangle=0,\left\langle i \bar{\psi} \gamma_{5} \psi\right\rangle \neq 0$ respectively in the strong coupling limit. The inclusion of higherorder contributions to the strong-coupling $1 / N$ expansions breaks the vacuum degeneracy by selecting the standard vacuum with $\left\langle i \bar{\psi} \gamma_{5} \tau_{3} \psi\right\rangle \neq 0$, but the normalized difference of vacuum energy densities of the two vacua is of order $10^{-14}$ at $\beta=2.0$ and $N=3$, showing the extremely high instability of the Aoki solution.

The second possible origin of the discrepancies between the non-standard scenario of $[21,34]$ and the $W \chi P T$ analysis of [11], the analysis of which will be the main subject of this paper, lies in the following point. The chiral effective Lagrangian approach is based, as it is well known, on the assumption that the relevant low-energy degrees of freedom in QCD are the three pions. This assumption can be reliable in the physical phase, up to the critical line, and also in the Aoki phase, near the critical line, but it could break down as we go deep in the Aoki phase, where the neutral pion is massive. Indeed, QCD with two degenerate flavors of Wilson fermions of bare mass $m_{0}=-4.0$ in lattice units should also show degenerate vacua with $\left\langle i \bar{\psi} \gamma_{5} \tau_{3} \psi\right\rangle \neq 0$, $\left\langle i \bar{\psi} \gamma_{5} \psi\right\rangle=0$ and $\left\langle i \bar{\psi} \gamma_{5} \tau_{3} \psi\right\rangle=0,\left\langle i \bar{\psi} \gamma_{5} \psi\right\rangle \neq 0$ respectively, as discussed in [34].

With the purpose of establishing the range of applicability of the standard QCD chiral effective Lagrangian approach, we will analyze in this paper the vacuum structure of a generalized Nambu-Jona-Lasinio model (NJL) with Wilson fermions in the mean-field or leading-order $1 / N$ expansion. The model has been chosen to possess the more general $S U(2)_{V} \times S U(2)_{A}$ symmetry in the continuum, in analogy to QCD. The election of the NJL model for our analysis was motivated by the fact that four-dimensional models without gauge fields, and with four-fermion interactions, are considered as effective models to describe the low-energy physics of $\mathrm{QCD}^{1}[35$, 36].

The outline of the paper is as follows. In Section 2 we describe the model in the continuum and its lattice regularized version with Wilson fermions, as well as the way in which the model can be analytically solved in the mean-field $1 / N$ expansion with the help of eight auxiliary scalar and pseudoscalar fields. The gap equations and the phase diagram of the mean-field model in the various physically relevant cases are analyzed in Section 3. In Section 4 we show how the mean-field equations of our generalized NJL model can be obtained in the leading order of the $1 / N$ expansion of a four-fermion model with non-trivial color and flavor interactions, but where the action is local and free from the sign problem. Section 5 summarizes our conclusions.

\section{The model}

The most general four-fermion continuum Lagrangian in Euclidean space with $S U(2)_{V} \times$ $S U(2)_{A}$ symmetry in the chiral limit and with trivial color dependence can be written as follows,

$$
-\mathcal{L}=-\bar{\psi}(\not \partial+m) \psi+G_{1}\left[(\bar{\psi} \psi)^{2}+\left(i \bar{\psi} \gamma_{5} \vec{\tau} \psi\right)^{2}\right]+G_{2}\left[\left(i \bar{\psi} \gamma_{5} \psi\right)^{2}+(\bar{\psi} \vec{\tau} \psi)^{2}\right],
$$

\footnotetext{
1 For a review on the NJL model, see [37] and references therein.
} 
where $\psi$ is a fermion field with four Dirac and two flavor components, and $\tau^{a}$ are the Pauli matrices acting in flavor space. It is customary, in order to avoid the sign problem and/or to perform a $1 / N$ expansion, to add another ("color") degree of freedom to the spinors, and to straightforwardly generalize the interaction by replacing $\bar{\psi} B \psi \rightarrow \sum_{i=1}^{N} \bar{\psi}_{i} B \psi_{i}$, where $B$ is any of the matrices appearing in Eq. (1). Although the interaction is not diagonal in color space, it will become so after a Hubbard-Stratonovich transformation, and moreover it will be the same for every color: for this reason we will call it diagonal and trivial in color, with a small abuse of terminology. In the following, we will refer to this straightforward generalization as the $N$-color model.

The NJL model given by action (1) enjoys the same $S U(2)_{V} \times S U(2)_{A}$ symmetry of QCD and it is an effective model to describe the low-energy physics of QCD [35]. This model, regularized on a hypercubic four-dimensional lattice with Wilson fermions, was analyzed in the $G_{2}=0$ limit and in the mean-field or first-order $1 / N$ expansion by Aoki et al. [5], who found a phase, for large values of $G_{1}$, in which both flavor symmetry and parity are spontaneously broken, in close analogy to lattice QCD with Wilson fermions. The qualitative results of Aoki et al. were also corroborated by Bitar and Vranas in [33], where they found, using numerical simulations, the existence of this parity-flavor broken phase in the two-color model.

The lattice action of the $N$-color model in the Wilson regularization can be written as $S=$ $S_{0}+S_{I}$, with the free part of the action being

$$
S_{0}=\sum_{x, y} \bar{\psi}_{x} \Delta_{x y} \psi_{y}
$$

where now $\psi$ is a fermion field with four Dirac, two flavor and $N$ color components, and where the Dirac-Wilson operator $\Delta$ is given by

$$
\Delta_{x y}=\frac{1}{2} \sum_{\mu=1}^{4}\left[\left(\gamma_{\mu}-r\right) \delta_{x+\hat{\mu}, y}-\left(\gamma_{\mu}+r\right) \delta_{x-\hat{\mu}, y}\right]+\left(4 r+m_{0}\right) \delta_{x y},
$$

with $r$ the Wilson parameter and $m_{0}$ the bare fermion mass. The interaction part is

$$
-S_{I}=\sum_{x} \frac{G_{1}}{N}\left[\left(\bar{\psi}_{x} \psi_{x}\right)^{2}+\left(\bar{\psi}_{x} i \gamma_{5} \vec{\tau} \psi_{x}\right)^{2}\right]+\frac{G_{2}}{N}\left[\left(\bar{\psi}_{x} i \gamma_{5} \psi_{x}\right)^{2}+\left(\bar{\psi}_{x} \vec{\tau} \psi_{x}\right)^{2}\right],
$$

were we have conveniently redefined the coupling constants. As it is well known, the Wilson term breaks explicitly the full chiral symmetry, and so only parity and vector symmetries are kept in the lattice regularization. The four-fermion action can be bilinearized by performing a Hubbard-Stratonovich transformation, which implies the introduction of eight scalar and pseudoscalar auxiliary fields as follows,

$$
S_{B}=N \sum_{x}\left[\beta_{1}\left(\sigma_{x}^{2}+\vec{\pi}_{x}^{2}\right)+\beta_{2}\left(\eta_{x}^{2}+\vec{\rho}_{x}^{2}\right)\right]+\sum_{x, y} \bar{\psi}_{x} M_{x y} \psi_{y},
$$

where the fermion matrix $M$ is

$$
M_{x y}=\Delta_{x y}+\delta_{x y}\left(\sigma_{x}+i \gamma_{5} \vec{\tau} \cdot \vec{\pi}_{x}+i \gamma_{5} \eta_{x}+\vec{\tau} \cdot \vec{\rho}_{x}\right),
$$

and moreover $\beta_{i}=1 /\left(4 G_{i}\right)$. Here we are considering the case $G_{1}, G_{2} \geqslant 0$.

In the $G_{2}=0$ case analyzed in [5,33] it is easy to see that the fermion determinant is real ${ }^{2}$ and therefore the theory, with an even number of colors, is free from the sign problem. This allows

\footnotetext{
${ }^{2}$ Reality is readily proved by noting that $C M C^{\dagger}=M^{*}$, with $C=\tau_{2} \gamma^{1} \gamma^{3}$ and $C C^{\dagger}=1$.
} 
to consistently perform the $1 / N$ expansion [5] and the numerical simulations in the two-color model [33]. Unfortunately, in the general case $\left(G_{1} \neq 0, G_{2} \neq 0\right)$ the fermion determinant is complex, and even if the leading order of the $1 / N$ expansion is free from the sign problem for an even number of colors, the very consistency of this expansion is, at least, doubtful. This is the reason why we decided to study the infinite range model, or mean-field approximation, where again one can easily show that the sign problem is absent for an even number of colors. However, in Section 4 we will show how the gap equations of the infinite range model are just the same obtained at leading order in the $1 / N$ expansion of a four-fermion model with local interactions, the same symmetries, and free from the sign problem.

\section{The phase diagram of the mean-field model}

The interaction part $S_{I}^{(\mathrm{MF})}$ of the lattice action $S^{(\mathrm{MF})}=S_{0}+S_{I}^{(\mathrm{MF})}$ for the infinite-range model can be written as follows,

$$
\begin{aligned}
-S_{I}^{(\mathrm{MF})}= & \frac{G_{1}}{N} \frac{1}{V}\left[\left(\sum_{x} \bar{\psi}_{x} \psi_{x}\right)^{2}+\left(\sum_{x} \bar{\psi}_{x} i \gamma_{5} \vec{\tau} \psi_{x}\right)^{2}\right] \\
& +\frac{G_{2}}{N} \frac{1}{V}\left[\left(\sum_{x} \bar{\psi}_{x} i \gamma_{5} \psi_{x}\right)^{2}+\left(\sum_{x} \bar{\psi}_{x} \vec{\tau} \psi_{x}\right)^{2}\right],
\end{aligned}
$$

where $V$ is the number of lattice sites. Performing again a Hubbard-Stratonovich transformation we get for the bilinearized action

$$
S_{B}^{(\mathrm{MF})}=V N\left[\beta_{1}\left(\sigma^{2}+\vec{\pi}^{2}\right)+\beta_{2}\left(\eta^{2}+\vec{\rho}^{2}\right)\right]+\sum_{x, y} \bar{\psi}_{x} M_{x y} \psi_{y},
$$

where the auxiliary fields are now constant fields, the fermion matrix is

$$
M_{x y}=\Delta_{x y}+\delta_{x y}\left(\sigma+i \gamma_{5} \vec{\tau} \cdot \vec{\pi}+i \gamma_{5} \eta+\vec{\tau} \cdot \vec{\rho}\right),
$$

and again $\beta_{i}=1 /\left(4 G_{i}\right)$, with $G_{1}, G_{2} \geqslant 0$. The integral over the fermion fields can again be done analytically and in the limit of large volume $V$ the model can be solved by writing down and solving the saddle-point equations.

Integrating out the fermionic degrees of freedom, the partition function of the mean-field model reads

$$
\begin{aligned}
Z & =\int d \sigma d^{3} \pi d \eta d^{3} \rho \operatorname{Det} M e^{-N V\left[\beta_{1}\left(\sigma^{2}+\vec{\pi}^{2}\right)+\beta_{2}\left(\eta^{2}+\vec{\rho}^{2}\right)\right]} \\
& \equiv \int d \sigma d^{3} \pi d \eta d^{3} \rho e^{-2 N V \mathcal{V}_{\mathrm{eff}}}
\end{aligned}
$$

with $\mathcal{V}_{\text {eff }}$ the effective potential per flavor and color. As we show in Appendix A, the fermionic determinant is real in this case; since we are taking an even number of colors, Det $M$ is also positive, so that there is no sign problem, and we can write Det $M=\left(\operatorname{Det} M M^{\dagger}\right)^{\frac{1}{2}}$.

In order to compute the determinant it is convenient to go over to momentum space. Starting from a finite lattice with periodic boundary conditions and then taking the limit of infinite volume, one obtains

$$
\operatorname{Det} M=\exp \left\{\frac{V}{2} \int_{B} \frac{d^{4} p}{(2 \pi)^{4}} \operatorname{tr} \log \tilde{M}(p) \tilde{M}(p)^{\dagger}+\mathcal{O}\left(V^{-1}\right)\right\},
$$


where

$$
M_{x y}=\int_{B} \frac{d^{4} p}{(2 \pi)^{4}} e^{-i p \cdot(x-y)} \tilde{M}(p), \quad \tilde{M}(p)=\sum_{x} e^{i p \cdot x} M_{x 0},
$$

and where $B$ is the first Brillouin zone $p_{\mu} \in[0,2 \pi], \mu=1, \ldots, 4$ (or equivalently $p_{\mu} \in[-\pi, \pi]$ due to periodicity), and tr stands for the trace over Dirac, flavor and color indices. A straightforward calculation shows that

$$
\tilde{M}(p)=i \sum_{\mu=1}^{4} \gamma_{\mu} \sin p_{\mu}+r\left(4-\sum_{\mu=1}^{4} \cos p_{\mu}\right)+m_{0}+\sigma+i \gamma_{5} \vec{\tau} \cdot \vec{\pi}+i \gamma_{5} \eta+\vec{\tau} \cdot \vec{\rho}
$$

The effective potential $\mathcal{V}_{\text {eff }}$ can be computed explicitly, and reads

$$
\mathcal{V}_{\text {eff }}=\frac{\beta_{1}}{2}\left(\sigma^{2}+\vec{\pi}^{2}\right)+\frac{\beta_{2}}{2}\left(\eta^{2}+\vec{\rho}^{2}\right)-\int_{B} \frac{d^{4} p}{(2 \pi)^{4}} \log Q
$$

with

$$
\begin{aligned}
Q= & \Sigma(p)^{2}+2 \Sigma(p)\left[\left(w_{r}(p)+m_{0}+\sigma\right)^{2}+\vec{\pi}^{2}+\left(\eta^{2}+\vec{\rho}^{2}\right)\right] \\
& +\left[\left(w_{r}(p)+m_{0}+\sigma\right)^{2}+\vec{\pi}^{2}-\left(\eta^{2}+\vec{\rho}^{2}\right)\right]^{2} \\
& +4\left[\eta\left(w_{r}(p)+m_{0}+\sigma\right)-\vec{\rho} \cdot \vec{\pi}\right]^{2},
\end{aligned}
$$

where we have set

$$
\Sigma(p)=\sum_{\mu=1}^{4}\left(\sin p_{\mu}\right)^{2}, \quad w_{r}(p)=r\left(4-\sum_{\mu=1}^{4} \cos p_{\mu}\right) .
$$

Notice that $Q \geqslant 0$.

In the large volume limit, the partition function will be dominated by the contribution coming from the minimum of the effective potential, and so it can be computed through the saddle-point technique. In order to look for the minimum of the effective potential, it is convenient to reorder the terms in Eq. (15). A little algebra allows to rewrite it as

$$
\begin{aligned}
Q= & {\left[\Sigma(p)+\left(w_{r}(p)+m_{0}+\sigma\right)^{2}+\Pi^{2}+\eta^{2}+\rho^{2}\right]^{2} } \\
& -4\left[\rho\left(w_{r}(p)+m_{0}+\sigma\right)+\Pi \eta \cos \theta\right]^{2}-4\left(\eta^{2}+\rho^{2}\right) \Pi^{2}(\sin \theta)^{2},
\end{aligned}
$$

where we have set

$$
\begin{aligned}
& \Pi=|\vec{\pi}|, \quad \rho=|\vec{\rho}|, \\
& \vec{\pi} \cdot \vec{\rho}=\Pi \rho \cos \theta .
\end{aligned}
$$

Setting also

$$
\begin{aligned}
& Q_{0}=\left[\Sigma(p)+\left(w_{r}(p)+m_{0}+\sigma\right)^{2}+\Pi^{2}+\eta^{2}+\rho^{2}\right]^{2} \geqslant 0, \\
& Q_{1}=4\left[\rho\left(w_{r}(p)+m_{0}+\sigma\right)+\Pi \eta \cos \theta\right]^{2}+4\left(\eta^{2}+\rho^{2}\right) \Pi^{2}(\sin \theta)^{2} \geqslant 0,
\end{aligned}
$$

we have $Q=Q_{0}-Q_{1}$, and the effective potential can be rewritten as

$$
\mathcal{V}_{\mathrm{eff}}=\frac{\beta_{1}}{2}\left(\sigma^{2}+\Pi^{2}\right)+\frac{\beta_{2}}{2}\left(\eta^{2}+\rho^{2}\right)-\int_{B} \frac{d^{4} p}{(2 \pi)^{4}}\left[\log Q_{0}+\log \left(1-\frac{Q_{1}}{Q_{0}}\right)\right] .
$$


It is convenient for our purposes to group the various terms in two different ways. The first way is

$$
\begin{aligned}
\mathcal{V}_{\text {eff }}= & \frac{\beta_{1}}{2}\left(\sigma^{2}+\Pi^{2}+\eta^{2}+\rho^{2}\right)-\int_{B} \frac{d^{4} p}{(2 \pi)^{4}} \log Q_{0} \\
& +\frac{\left(\beta_{2}-\beta_{1}\right)}{2}\left(\eta^{2}+\rho^{2}\right)-\int_{B} \frac{d^{4} p}{(2 \pi)^{4}} \log \left(1-\frac{Q_{1}}{Q_{0}}\right),
\end{aligned}
$$

which as we will see is appropriate for the case $\beta_{1}<\beta_{2}$, and the second way is

$$
\begin{aligned}
\mathcal{V}_{\text {eff }}= & \frac{\beta_{1}}{2} \sigma^{2}+\frac{\beta_{2}}{2}\left(\Pi^{2}+\eta^{2}+\rho^{2}\right)-\int_{B} \frac{d^{4} p}{(2 \pi)^{4}} \log Q_{0} \\
& +\frac{\left(\beta_{1}-\beta_{2}\right)}{2} \Pi^{2}-\int_{B} \frac{d^{4} p}{(2 \pi)^{4}} \log \left(1-\frac{Q_{1}}{Q_{0}}\right),
\end{aligned}
$$

which is appropriate for the case $\beta_{1}>\beta_{2}$. The key observation is that $Q_{0}$ depends only on $\sigma$ and on the combination $z^{2} \equiv \Pi^{2}+\eta^{2}+\rho^{2}$, so that the first line in both equations depends only on $\sigma$ and $z$. Therefore, the minimization of the effective potential at fixed $\sigma$ and $z$ involves only the second line of Eqs. (21) and (22).

To make things more transparent, let us introduce the new set of variables $z, \omega, \varphi$, in terms of which one writes

$$
\begin{aligned}
& \Pi=z \cos \omega, \\
& \eta=z \sin \omega \cos \varphi, \\
& \rho=z \sin \omega \sin \varphi .
\end{aligned}
$$

The range of these variables is $z \geqslant 0, \omega \in\left[0, \frac{\pi}{2}\right], \varphi \in[0, \pi]$, that corresponds to the range $\Pi \geqslant 0$, $\rho \geqslant 0, \eta \in \mathbb{R}$ of the original variables. In terms of the new variables, Eq. (21) reads

$$
\begin{aligned}
\mathcal{V}_{\mathrm{eff}}(\sigma, z, \omega, \varphi)= & \frac{\beta_{1}}{2}\left(\sigma^{2}+z^{2}\right)-\int_{B} \frac{d^{4} p}{(2 \pi)^{4}} \log Q_{0}(\sigma, z) \\
& -\frac{\Delta \beta}{2}(z \sin \omega)^{2}-\int_{B} \frac{d^{4} p}{(2 \pi)^{4}} \log \left(1-\frac{Q_{1}(\sigma, z, \omega, \varphi)}{Q_{0}(\sigma, z)}\right),
\end{aligned}
$$

where $\Delta \beta \equiv \beta_{1}-\beta_{2}$ and we have made explicit the dependence on the relevant variables, and Eq. (22) reads

$$
\begin{aligned}
\mathcal{V}_{\text {eff }}(\sigma, z, \omega, \varphi)= & \frac{\beta_{1}}{2} \sigma^{2}+\frac{\beta_{2}}{2} z^{2}-\int_{B} \frac{d^{4} p}{(2 \pi)^{4}} \log Q_{0}(\sigma, z) \\
& +\frac{\Delta \beta}{2}(z \cos \omega)^{2}-\int_{B} \frac{d^{4} p}{(2 \pi)^{4}} \log \left(1-\frac{Q_{1}(\sigma, z, \omega, \varphi)}{Q_{0}(\sigma, z)}\right) .
\end{aligned}
$$

As we have already noted, the first line in Eqs. (24) and (25) depends only on $\sigma$ and $z$. As a consequence, in order to minimize the effective potential with respect to $\omega$ and $\varphi$ we have to 
focus on the second line only. Moreover, since $Q \geqslant 0$, we have that $Q_{1} \leqslant Q_{0}$, and so the last term in Eqs. (24) and (25) is positive or zero,

$$
\Delta \mathcal{V} \equiv-\int_{B} \frac{d^{4} p}{(2 \pi)^{4}} \log \left(1-\frac{Q_{1}(\sigma, z, \omega, \varphi)}{Q_{0}(\sigma, z)}\right) \geqslant 0 .
$$

Therefore, the second line in Eq. (24) is positive or zero if $\beta_{2} \geqslant \beta_{1}$, and the second line in Eq. (25) is positive or zero if $\beta_{1} \geqslant \beta_{2}$; in particular, both terms are positive or zero. If we can find values of $\omega$ and $\varphi$ such that these lower bounds are saturated, then we have automatically minimized the effective potential with respect to $\omega$ and $\varphi$. In order to do so, we need to make both terms vanish, and in particular we need that $Q_{1}$ vanishes identically as a function of the momentum. ${ }^{3}$ In terms of our new variables, $Q_{1}$ reads

$$
Q_{1}=4(z \sin \omega)^{2}\left\{(z \cos \omega \sin \theta)^{2}+\left[\sin \varphi\left(w_{r}(p)+m_{0}+\sigma\right)+z \cos \omega \cos \varphi \cos \theta\right]^{2}\right\} .
$$

One sees immediately that $Q_{1}$ vanishes identically if $z=0$, or if $\sin \omega=0$, i.e., $\omega=0$. If $z \neq 0$, $\omega \neq 0$, then both terms in braces must be zero, and the second one must be so independently of $p$ : this can happen only if $\sin \varphi=0$, i.e., $\varphi=0, \pi$, which in turn requires that $\cos \omega=0$, i.e., $\omega=\frac{\pi}{2}$.

Summarizing, $Q_{1}$ vanishes identically only if ${ }^{4}$

1. $\omega=0$;

2. $\omega=\frac{\pi}{2}, \quad \varphi=0, \pi$,

independently of the values of $\sigma$ and $z$. Stated differently, in terms of the original variables, $Q_{1}$ vanishes identically only if

$$
\begin{array}{lll}
\text { 1. } & \rho=0, & |\eta|=0 ; \\
\text { 2. } & \rho=0, & \Pi=0 .
\end{array}
$$

It is immediate to check that in case 1 the second line of Eq. (24) vanishes, while in case 2 the second line of Eq. (25) vanishes, independently of $\Delta \beta$. Let us now discuss the various cases separately.

Case $\boldsymbol{\beta}_{\mathbf{1}}<\boldsymbol{\beta}_{\mathbf{2}}$. In this case, the minimum of the effective potential lies on the curve $\omega=0$, and is obtained by minimizing the functional

$$
\mathcal{V}_{<}=\frac{\beta_{1}}{2}\left(\sigma^{2}+z^{2}\right)-\int_{B} \frac{d^{4} p}{(2 \pi)^{4}} \log Q_{0}(\sigma, z),
$$

with respect to $\sigma$ and $z$. Since $\omega=0$ corresponds to $\eta=\rho=0$, in this case $z=\Pi$. Clearly, the minimum will be independent of $\beta_{2}$. The gap equations read therefore

\footnotetext{
3 In principle it is sufficient that $Q_{1}$ is nonzero only on a set of zero measure in the four-dimensional momentum space, but it is easy to see that either $Q_{1}$ vanishes identically or it vanishes on a three-dimensional hypersurface.

4 Roughly speaking, since at $z=0$ all values of $\omega$ and $\varphi$ are equivalent, these two cases include also the case $z=0$.
} 


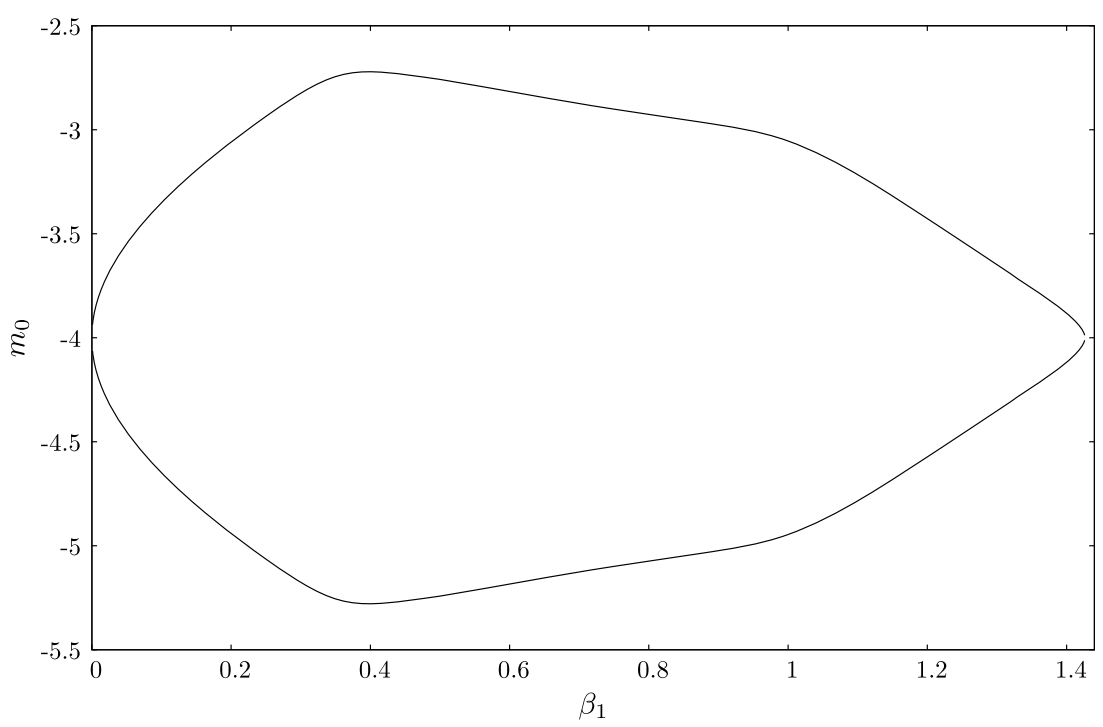

Fig. 1. Boundary of the phase with broken parity and flavor for $\Delta \beta=\beta_{1}-\beta_{2}<0$ in the $\left(\beta_{1}, m_{0}\right)$ plane. When $\Delta \beta=0$, i.e., $\beta_{1}=\beta_{2}$, two degenerate vacua with broken parity exist, one with broken and one with unbroken flavor symmetry. Here we set $r=1$.

$$
\begin{aligned}
& 0=\frac{\beta_{1}}{4} \sigma-\int_{B} \frac{d^{4} p}{(2 \pi)^{4}} \frac{w_{r}(p)+m_{0}+\sigma}{\Sigma(p)+\left(w_{r}(p)+m_{0}+\sigma\right)^{2}+\Pi^{2}}, \\
& 0=\left[\frac{\beta_{1}}{4}-\int_{B} \frac{d^{4} p}{(2 \pi)^{4}} \frac{1}{\Sigma(p)+\left(w_{r}(p)+m_{0}+\sigma\right)^{2}+\Pi^{2}}\right] \Pi .
\end{aligned}
$$

There are two solutions to these equations. The first one has $\Pi=0$ and $\sigma$ determined by the solution to the equation

$$
\frac{\beta_{1}}{4} \sigma=\int_{B} \frac{d^{4} p}{(2 \pi)^{4}} \frac{w_{r}(p)+m_{0}+\sigma}{\Sigma(p)+\left(w_{r}(p)+m_{0}+\sigma\right)^{2}},
$$

which always exists. ${ }^{5}$ A second solution with nonzero $\Pi$ is obtained by solving

$$
\begin{aligned}
& 0=\int_{B} \frac{d^{4} p}{(2 \pi)^{4}} \frac{w_{r}(p)+m_{0}}{\Sigma(p)+\left(w_{r}(p)+m_{0}+\sigma\right)^{2}+\Pi^{2}}, \\
& \frac{\beta_{1}}{4}=\int_{B} \frac{d^{4} p}{(2 \pi)^{4}} \frac{1}{\Sigma(p)+\left(w_{r}(p)+m_{0}+\sigma\right)^{2}+\Pi^{2}} .
\end{aligned}
$$

5 To see this it is enough to show that the right-hand side is always finite, and that it vanishes as $1 / \sigma$ for large $|\sigma|$. The second point is trivial, while to prove the first one it is enough to bound the right-hand side as follows:

$$
\int_{B} \frac{d^{4} p}{(2 \pi)^{4}} \frac{w_{r}(p)+m_{0}+\sigma}{\Sigma(p)+\left(w_{r}(p)+m_{0}+\sigma\right)^{2}} \leqslant\left(8+m_{0}+\sigma\right) \int_{B} \frac{d^{4} p}{(2 \pi)^{4}} \frac{1}{\Sigma(p)}<\infty .
$$




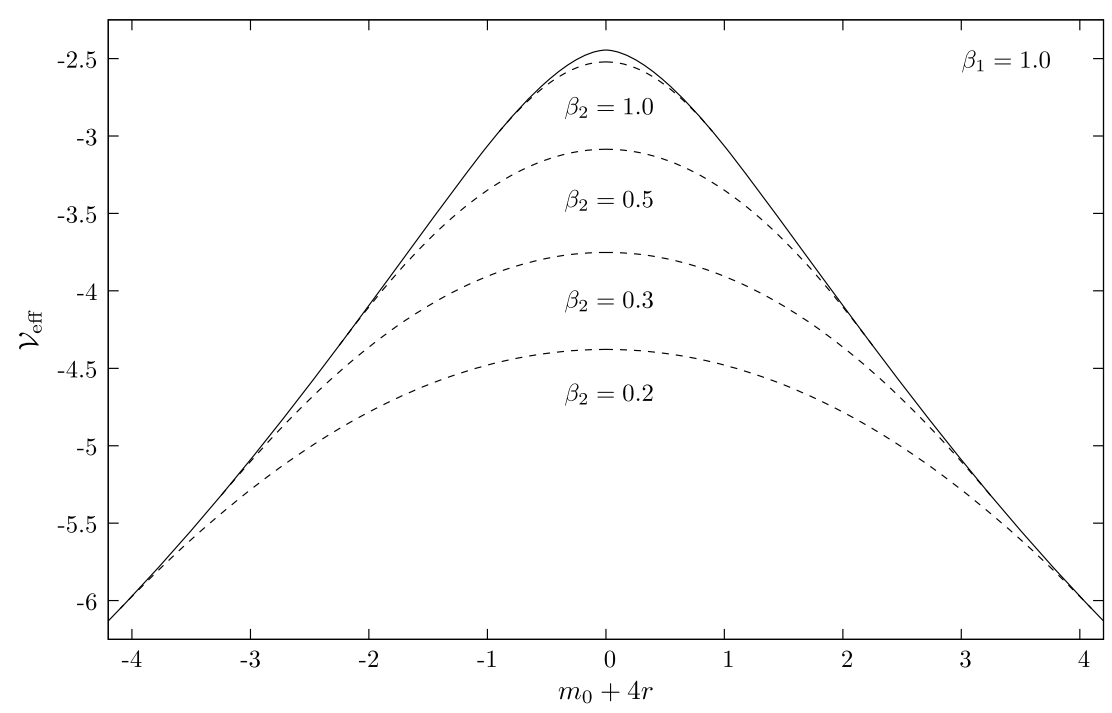

Fig. 2. Effective potential for the solution with $\eta=0$, Eq. (32) (solid line), and for the solution with $\eta \neq 0$, Eq. (36), in the case $\beta_{1}=1.0$ and for various values of $\beta_{2} \leqslant \beta_{1}$. Here we set $r=1$.

This solution is clearly degenerate since only the modulus of $\vec{\pi}$ is determined, while the direction is arbitrary on the sphere $S^{2}$. As it has been shown in Ref. [5], a solution to Eq. (33) exists only in a closed and bounded region in the $\left(\beta_{1}, m_{0}\right)$ plane (see Fig. 1); on the other hand, when it exists it also minimizes the effective potential. Therefore, inside the region enclosed in the solid line in Fig. 1 , we have a phase with $\Pi \neq 0$, so that $\left\langle\bar{\psi} i \gamma_{5} \vec{\tau} \psi\right\rangle=-2 N \beta_{1} \vec{\pi} \neq 0$, and thus parity and flavor are broken. On the other hand, $\left\langle\bar{\psi} i \gamma_{5} \psi\right\rangle=-2 N \beta_{2} \eta=0$, so that the vacuum is of the standard Aoki type.

Case $\boldsymbol{\beta}_{\mathbf{1}}>\boldsymbol{\beta}_{\mathbf{2}}$. In this case, the minimum of the effective potential lies on the curves $\omega=\frac{\pi}{2}$, $\varphi=0, \pi$, and is obtained by minimizing the functional

$$
\mathcal{V}_{>}=\frac{\beta_{1}}{2} \sigma^{2}+\frac{\beta_{2}}{2} z^{2}-\int_{B} \frac{d^{4} p}{(2 \pi)^{4}} \log Q_{0}(\sigma, z)
$$

with respect to $\sigma$ and $z$. Since $\omega=\frac{\pi}{2}, \varphi=0, \pi$, corresponds to $\Pi=\rho=0$, in this case $z=|\eta|$. Notice that $\varphi=0$ and $\varphi=\pi$ give the same $\mathcal{V}_{>}$. The gap equations read therefore

$$
\begin{aligned}
& 0=\frac{\beta_{1}}{4} \sigma-\int_{B} \frac{d^{4} p}{(2 \pi)^{4}} \frac{w_{r}(p)+m_{0}+\sigma}{\Sigma(p)+\left(w_{r}(p)+m_{0}+\sigma\right)^{2}+\eta^{2}}, \\
& 0=\left[\frac{\beta_{2}}{4}-\int_{B} \frac{d^{4} p}{(2 \pi)^{4}} \frac{1}{\Sigma(p)+\left(w_{r}(p)+m_{0}+\sigma\right)^{2}+\eta^{2}}\right]|\eta| .
\end{aligned}
$$

There are two solutions to these equations, one with $|\eta|=0$ and $\sigma$ determined by the solution to Eq. (32), which always exists, and a second solution with nonzero $|\eta|$, obtained by solving 


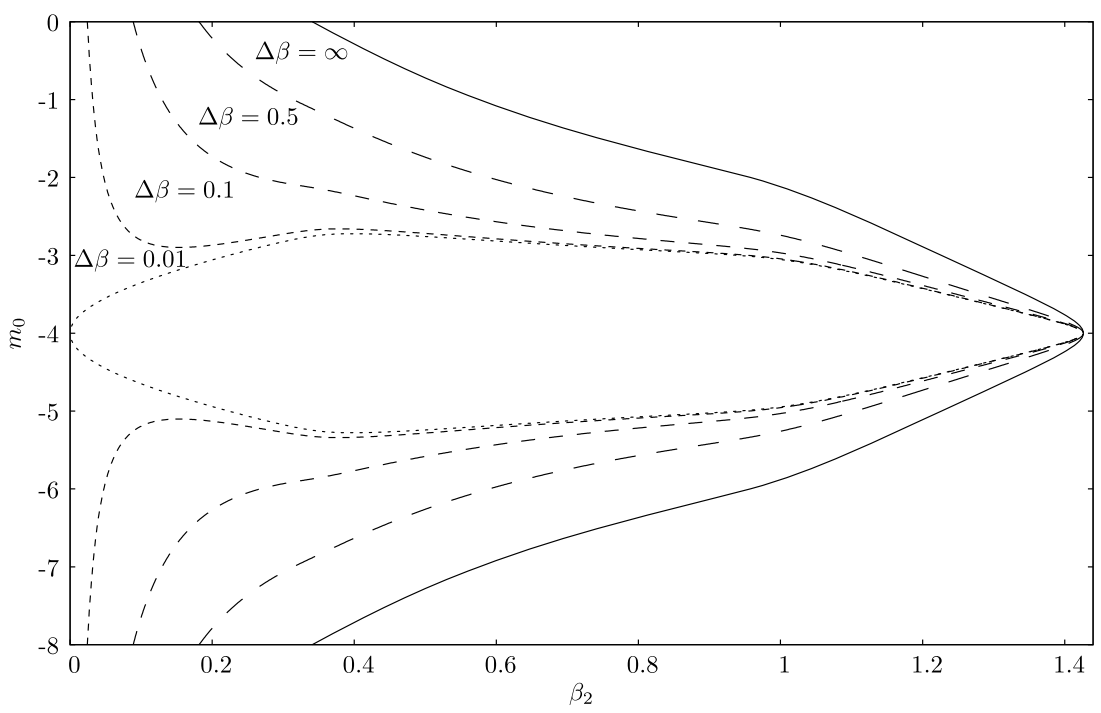

Fig. 3. Boundary of the phase with broken parity for various finite values of $\Delta \beta=\beta_{1}-\beta_{2}>0$ in the $\left(\beta_{2}, m_{0}\right)$ plane (dashed lines). The solid line corresponds to the case $\Delta \beta=\infty$, i.e., $G_{1}=0$. The dotted line corresponds to the case $\Delta \beta=0$, see Fig. 1, where two degenerate vacua with broken parity exist, one with broken and one with unbroken flavor symmetry. Here we set $r=1$.

$$
\begin{aligned}
& \frac{\Delta \beta}{4} \sigma=\int_{B} \frac{d^{4} p}{(2 \pi)^{4}} \frac{w_{r}(p)+m_{0}}{\Sigma(p)+\left(w_{r}(p)+m_{0}+\sigma\right)^{2}+\eta^{2}}, \\
& \frac{\beta_{2}}{4}=\int_{B} \frac{d^{4} p}{(2 \pi)^{4}} \frac{1}{\Sigma(p)+\left(w_{r}(p)+m_{0}+\sigma\right)^{2}+\eta^{2}},
\end{aligned}
$$

that exists only in a certain region of the parameter space. This solution is (at least) twofold degenerate, since only the absolute value of $\eta$ is determined, while the sign is not.

Numerical investigations show that the solution to Eq. (36), when it exists, is unique (up to the sign of $\eta$ ) and yields the absolute minimum of the effective potential, see Fig. 2. In particular, for any choice of $\Delta \beta=\beta_{1}-\beta_{2}>0$, there is a region in the $\left(\beta_{2}, m_{0}\right)$ plane where the solution exists and minimizes the potential, see Fig. 3. This region is symmetric with respect to $m_{0}=-4 r$, and does not extend beyond $\beta=\beta_{c} \simeq 1.43057$. This region corresponds to a phase where the condensate $\left\langle\bar{\psi} i \gamma_{5} \psi\right\rangle=-2 N \beta_{2} \eta \neq 0$, so that parity is broken; on the other hand, since $\Pi=$ $\rho=0$, flavor is not broken. The vacuum is therefore not of the standard Aoki type. Notice that $\eta=0$ on the phase boundary.

Case $\boldsymbol{\beta}_{\mathbf{1}}=\boldsymbol{\beta}_{\mathbf{2}} \equiv \boldsymbol{\beta}$. In this case there are two degenerate sets of minima, one with $\omega=0$, and one with $\omega=\frac{\pi}{2}, \varphi=0, \pi$, both obtained by minimizing the functional

$$
\mathcal{V}_{=}=\frac{\beta}{2}\left(\sigma^{2}+z^{2}\right)-\int_{B} \frac{d^{4} p}{(2 \pi)^{4}} \log Q_{0}(\sigma, z)
$$

with respect to $\sigma$ and $z$. The minimum at $\omega=0$ corresponds to $\eta=\rho=0$ and $z=\Pi$, while the minima at $\omega=\frac{\pi}{2}, \varphi=0, \pi$ correspond to $\Pi=\rho=0$ and $z=|\eta|$. The gap equations read therefore 


$$
\begin{aligned}
& 0=\frac{\beta}{4} \sigma-\int_{B} \frac{d^{4} p}{(2 \pi)^{4}} \frac{w_{r}(p)+m_{0}+\sigma}{\Sigma(p)+\left(w_{r}(p)+m_{0}+\sigma\right)^{2}+z^{2}}, \\
& 0=\left[\frac{\beta}{4}-\int_{B} \frac{d^{4} p}{(2 \pi)^{4}} \frac{1}{\Sigma(p)+\left(w_{r}(p)+m_{0}+\sigma\right)^{2}+z^{2}}\right] z .
\end{aligned}
$$

There are two solutions to these equations, one with $z=0$ and $\sigma$ determined by solving Eq. (32), which can always be done, and a second set of solutions with nonzero $z$, obtained by solving

$$
\begin{aligned}
& 0=\int_{B} \frac{d^{4} p}{(2 \pi)^{4}} \frac{w_{r}(p)+m_{0}}{\Sigma(p)+\left(w_{r}(p)+m_{0}+\sigma\right)^{2}+z^{2}}, \\
& \frac{\beta}{4}=\int_{B} \frac{d^{4} p}{(2 \pi)^{4}} \frac{1}{\Sigma(p)+\left(w_{r}(p)+m_{0}+\sigma\right)^{2}+z^{2}},
\end{aligned}
$$

which is possible in a certain region of the $\left(\beta, m_{0}\right)$ plane. Setting $\eta=\rho=0$ and $z=\Pi$, there is a $S^{2}$ degeneracy, while setting $\Pi=\rho=0$ and $z=|\eta|$ there is a twofold degeneracy. Clearly, since this equation is identical to Eq. (33), up to the substitutions $\Pi \rightarrow z$ and $\beta_{1} \rightarrow \beta$, a region exists in the $\left(\beta, m_{0}\right)$ plane where the absolute minimum lies at nonzero $z$, which coincides with the one found by Aoki and collaborators, see Fig. 1. In this case, however, there are two degenerate (sets of) vacua with broken parity, one with broken and one with unbroken flavor symmetry, that are not connected by a symmetry transformation. In this case we have the coexistence of standard Aoki and non-standard Aoki vacua.

\section{A model with local interactions}

In this section we describe a $N$-color model with local interactions, free from the sign problem, which in the limit of large $N$ has the same phase diagram of the mean-field theory, discussed in the previous section. The action of the model is $S^{(\text {loc })}=S_{0}+S_{I}^{(\text {loc })}$, with the free part of the action being given in Eqs. (2) and (3), and the interaction part being

$$
-S_{I}^{\text {(loc) }}=\sum_{x} \frac{G_{1}}{N}\left[\left(\bar{\psi}_{x} \psi_{x}\right)^{2}+\left(\bar{\psi}_{x} i \gamma_{5} \vec{\tau} \psi_{x}\right)^{2}\right]+\frac{G_{2}}{N}\left[\left(\bar{\psi}_{x} i \gamma_{5} \lambda \psi_{x}\right)^{2}+\left(\bar{\psi}_{x} \vec{\tau} \lambda \psi_{x}\right)^{2}\right],
$$

where we are taking an even number of colors $N$, and where the $N \times N$ diagonal matrix $\lambda$ acting in color space is

$$
\lambda=\operatorname{diag}(1,-1,1,-1, \ldots, 1,-1) .
$$

After a Hubbard-Stratonovich transformation, the partition function takes the form

$$
\begin{aligned}
Z & =\int \mathcal{D} \sigma \mathcal{D} \vec{\pi} \mathcal{D} \eta \mathcal{D} \vec{\rho} \operatorname{Det} \bar{M} e^{-N\left[\sum_{x} \beta_{1}\left(\sigma_{x}^{2}+\vec{\pi}_{x}^{2}\right)+\beta_{2}\left(\eta_{x}^{2}+\vec{\rho}_{x}^{2}\right)\right]} \\
& \equiv \int \mathcal{D} \sigma \mathcal{D} \vec{\pi} \mathcal{D} \eta \mathcal{D} \vec{\rho} e^{-S_{\mathrm{eff}}}
\end{aligned}
$$

where now the fermion matrix is

$$
\bar{M}_{x y}=\Delta_{x y}+\delta_{x y}\left(\sigma_{x}+i \gamma_{5} \vec{\tau} \cdot \vec{\pi}_{x}+i \gamma_{5} \lambda \eta_{x}+\lambda \vec{\tau} \cdot \vec{\rho}_{x}\right) .
$$


It is easy to show that there is no sign problem in this case. Indeed, in color space the fermion matrix has the form

$$
\begin{aligned}
& \bar{M}_{x y}=\operatorname{diag}\left(M_{x y}, M_{x y}^{\prime}, M_{x y}, M_{x y}^{\prime}, \ldots, M_{x y}, M_{x y}^{\prime}\right), \\
& M_{x y}=\Delta_{x y}+\delta_{x y}\left(\sigma_{x}+i \gamma_{5} \vec{\tau} \cdot \vec{\pi}_{x}+i \gamma_{5} \eta_{x}+\vec{\tau} \cdot \vec{\rho}_{x}\right), \\
& M_{x y}^{\prime}=\Delta_{x y}+\delta_{x y}\left(\sigma_{x}+i \gamma_{5} \vec{\tau} \cdot \vec{\pi}_{x}-i \gamma_{5} \eta_{x}-\vec{\tau} \cdot \vec{\rho}_{x}\right),
\end{aligned}
$$

and one easily sees that $M_{x y}^{\prime}=C^{\dagger} M_{x y}^{*} C$, with $C=\gamma^{1} \gamma^{3} \tau_{2}$. Therefore,

$$
\operatorname{Det} \bar{M}=\left(\operatorname{Det} M \operatorname{Det} M^{\prime}\right)^{\frac{N}{2}}=\left(\operatorname{Det} M \operatorname{Det} M^{*}\right)^{\frac{N}{2}}=|\operatorname{Det} M|^{N} \text {, }
$$

which is clearly real and positive. We can therefore write for the effective action

$$
S_{\text {eff }}=\sum_{x} \beta_{1}\left(\sigma_{x}^{2}+\vec{\pi}^{2}\right)+\beta_{2}\left(\eta_{x}^{2}+\vec{\rho}_{x}^{2}\right)-\frac{1}{2}\left(\operatorname{Tr} \log M+\operatorname{Tr} \log M^{\dagger}\right),
$$

where $\operatorname{Tr}$ is here the trace over spacetime, Dirac and flavor indices.

In the limit of large $N$, we can parameterize the auxiliary fields as $\phi_{x}^{i}=\phi^{i}+\frac{\delta \phi_{x}^{i}}{\sqrt{N}}$, with space-independent values $\phi^{i}$, to be determined by the saddle-point equations $\frac{\delta \mathcal{V}_{\text {eff }}}{\delta \phi_{x}}=0$, where we have denoted collectively $\left\{\phi_{x}^{i}\right\}=\left(\sigma_{x}, \vec{\pi}_{x}, \eta_{x}, \vec{\rho}_{x}\right)$. Expanding the effective action with respect to $\delta \phi_{x}^{i}$ we find

$$
S_{\text {eff }}\left(\phi_{x}\right)=S_{\text {eff }}(\phi)+\frac{1}{2 N} \sum_{x, y, i, j} \delta \phi_{x}^{i} \frac{\delta^{2} S_{\text {eff }}}{\delta \phi_{x}^{i} \delta \phi_{y}^{j}} \delta \phi_{y}^{j}+\cdots
$$

Going over to momentum space, and taking the limit of large volume, we have

$$
S_{\text {eff }}\left(\phi_{x}\right)=2 N V \mathcal{V}_{\text {eff }}(\phi)+\frac{1}{2} \int_{B} \frac{d^{4} q}{(2 \pi)^{4}} \delta \tilde{\phi}(-q) G_{i j}^{-1}(q) \delta \tilde{\phi}(q)+\mathcal{O}\left(N^{-1}\right),
$$

where we have made explicit the dependence on $N$. The effective potential at the saddle point reads

$$
\mathcal{V}_{\mathrm{eff}}=\frac{\beta_{1}}{2}\left(\sigma^{2}+\vec{\pi}^{2}\right)+\frac{\beta_{2}}{2}\left(\eta^{2}+\vec{\rho}^{2}\right)-\int_{B} \frac{d^{4} p}{(2 \pi)^{4}} \log Q,
$$

with $Q$ given in Eq. (15), i.e., the same result (14) obtained in the mean-field case. Obviously, the phase diagram at large $N$ of the local model discussed here is therefore exactly the same found for the infinite-range model.

The first correction is expressed through the matrix

$$
\begin{aligned}
G_{i j}^{-1}(q)= & 2 \delta_{i j} \beta_{n_{i}}+\int_{B} \frac{d^{4} p}{(2 \pi)^{4}} \frac{1}{g\left(p+\frac{q}{2}, z\right) g\left(p-\frac{q}{2}, z\right)} \\
& \times \operatorname{tr}\left[\mathcal{M}_{i} \tilde{M}^{\dagger}\left(p+\frac{q}{2}\right) \mathcal{M}_{j} \tilde{M}^{\dagger}\left(p-\frac{q}{2}\right)\right],
\end{aligned}
$$

where $i, j=\sigma, \vec{\pi}, \eta, \vec{\rho}$, and moreover $n_{\sigma}=n_{\vec{\pi}}=1, n_{\eta}=n_{\vec{\rho}}=2$, the matrices $\mathcal{M}_{i}$ are

$$
\mathcal{M}_{\sigma}=1, \quad \mathcal{M}_{\vec{\pi}}=i \gamma_{5} \vec{\tau}, \quad \mathcal{M}_{\eta}=i \gamma_{5}, \quad \mathcal{M}_{\vec{\rho}}=\vec{\tau},
$$


and the matrix $\tilde{M}(p)$ is given in Eq. (13). The function $g(p, z)$ is given by

$$
g(p, z)=\Sigma(p)+\left(w_{r}(p)+m_{0}+\sigma\right)^{2}+z^{2},
$$

with $z=0$ in the unbroken phase, $z=|\vec{\pi}|$ in the standard Aoki phase, and $z=\eta$ in the nonstandard Aoki phase. The nonzero elements of the inverse propagator $G_{i j}^{-1}(q)$ in the various phases are given in Appendix B. It is interesting to check the values in the broken phases of the masses of the excitations corresponding to $\vec{\pi}$ and $\eta$, defined through the small- $q$ behavior of the corresponding diagonal terms of the inverse propagator. ${ }^{6}$ Choosing for convenience $\pi_{a}=\Pi \delta_{a 3}$, we set

$$
\begin{aligned}
& G_{\pi_{a} \pi_{a}}^{-1}(q)={ }_{q \rightarrow 0} Z_{\pi_{a}}^{-1}\left(q^{2}+m_{\pi_{a}}^{2}\right), \\
& G_{\eta \eta}^{-1}(q)={ }_{q \rightarrow 0} Z_{\eta}^{-1}\left(q^{2}+m_{\eta}^{2}\right),
\end{aligned}
$$

where $q^{2}=\sum_{\mu=1}^{4} q_{\mu}^{2}$. Using the results reported in Appendix B, we find that ${ }^{7}$

$$
\begin{aligned}
& m_{\pi_{3}}^{2}=\frac{2 \Pi^{2} I_{2}(0, \sigma, \Pi)}{I_{0}^{(2)}(\sigma, \Pi)-2 \Pi^{2} I_{2}^{(2)}(\sigma, \Pi)}, \quad m_{\pi_{1,2}}^{2}=0, \\
& m_{\eta}^{2}=\frac{\frac{\beta_{2}-\beta_{1}}{4}+2 \Pi^{2} I_{2}(0, \sigma, \Pi)}{I_{0}^{(2)}(\sigma, \Pi)-2 \Pi^{2} I_{2}^{(2)}(\sigma, \Pi)},
\end{aligned}
$$

in the standard Aoki phase $(\Pi \neq 0, \eta=0)$, and

$$
\begin{aligned}
& m_{\pi_{a}}^{2}=\frac{\frac{\beta_{1}-\beta_{2}}{4}+2 \eta^{2} I_{2}(0, \sigma, \eta)}{I_{0}^{(2)}(\sigma, \eta)-2 \eta^{2} I_{2}^{(2)}(\sigma, \eta)}, \\
& m_{\eta}^{2}=\frac{2 \eta^{2} I_{2}(0, \sigma, \eta)}{I_{0}^{(2)}(\sigma, \eta)-2 \eta^{2} I_{2}^{(2)}(\sigma, \eta)},
\end{aligned}
$$

in the non-standard Aoki phase $(\Pi=0, \eta \neq 0)$. The quantities $I_{0,2}(q, \sigma, z)=I_{0,2}(0, \sigma, z)-$ $q^{2} I_{0,2}^{(2)}(\sigma, z)+\mathcal{O}\left(q^{4}\right)$ are defined in Appendix B. Let us make a few remarks.

- In the "physical" phase, where parity and flavor symmetries are realized in the vacuum, the three pions and the $\eta$ are degenerate. The pion degeneracy follows from flavor symmetry, but the fact that the $\eta$ is degenerate with the three pions is probably an artifact of the large- $N$ mean-field approximation. The three pions and the $\eta$, on the other hand, become massless on the phase boundary.

- In the standard Aoki phase $\left(G_{1}>G_{2}\right)$ the "charged" pions are massless and the neutral pion and the $\eta$ are massive and degenerate, except at the phase boundary where all of them become massless.

- In the non-standard Aoki phase $\left(G_{1}<G_{2}\right)$ the three pions and the $\eta$ are massive and degenerate, and all of them become massless on the phase boundary.

\footnotetext{
6 In the broken phases, the $\vec{\pi}$ 's and the $\eta$ are not the physical states, since they mix respectively with $\sigma$ and $\vec{\rho}$ in the standard Aoki phase, and with $\vec{\rho}$ and $\sigma$ in the non-standard Aoki phase.

7 Although we have not been able to prove it in the general case, the denominators in Eqs. (54) and (55) are certainly positive at small and at large $\Pi$ or $\eta$; furthermore, we have checked numerically that they are positive in the whole broken phase.
} 
- In the special case $G_{1}=G_{2}$, the $\eta$ and the neutral pion are massive and degenerate in any of the two vacua, and again become massless at the phase boundary.

\section{Conclusions}

With the aim of establishing the range of applicability of the standard QCD chiral effective Lagrangian approach, we have analyzed in this paper the vacuum structure of a generalized Nambu-Jona-Lasinio model (NJL) with Wilson fermions in the mean-field or leading-order $1 / N$ expansion. The model has been chosen to possess the more general $S U(2)_{V} \times S U(2)_{A}$ symmetry in the continuum. The election of the NJL model for our analysis was motivated by the fact that four-dimensional models without gauge fields, and with four-fermion interactions, are considered as effective models to describe the low-energy physics of QCD.

This generalized NJL model shows a rich phase structure in the three-parameter space (two four-fermion couplings plus the bare fermion mass). Indeed, in addition to the standard "physical" phases in which both, parity and flavor symmetries are realized in the vacuum, new phases where flavor and/or parity are spontaneously broken are found. These phases are, for $G_{1}>G_{2}$, of the standard Aoki type with $\left\langle i \bar{\psi} \gamma_{5} \tau_{3} \psi\right\rangle \neq 0$, and $\left\langle i \bar{\psi} \gamma_{5} \psi\right\rangle=0$, in agreement also with predictions of the chiral effective Lagrangian approach. However when $G_{1}<G_{2}$ the new phase is characterized by $\left\langle i \bar{\psi} \gamma_{5} \tau_{3} \psi\right\rangle=0$ and $\left\langle i \bar{\psi} \gamma_{5} \psi\right\rangle \neq 0$. In the special case $G_{1}=G_{2}$, where the continuum NJL action has, in the chiral limit, a $U(2)_{V} \times U(2)_{A}$ symmetry, as QCD, two degenerate vacua, not connected by parity-flavor symmetry transformations, with broken parity coexist, one with broken and another one with unbroken flavor symmetry.

These results show how the phase structure of an effective model for low-energy QCD cannot be entirely understood from Wilson Chiral Perturbation Theory, based on the standard QCD chiral effective Lagrangian approach. Nonetheless, and as explained in [21,23-25,34], our conclusions leave no practical consequences for lattice practitioners: Wilson Chiral Perturbation Theory is still applicable outside the Aoki phase, and the properties of the "physical" phase of lattice QCD with Wilson fermions remain unchanged.

We have also shown in Section 4 that the mean-field equations of our generalized NJL model can be obtained in the leading order of the $1 / N$ expansion of a four-fermion model with nontrivial color and flavor interactions, and the same NJL-symmetries, but where the action is local and free from the sign problem, thus allowing to compute corrections to the $1 / N$ expansion and to perform numerical simulations at finite $N$ in order to test the stability of the mean-field results reported here.

\section{Acknowledgements}

This work was funded by an INFN-MICINN Collaboration (under grant AIC-D-2011-0663), MICINN (under grants FPA2009-09638 and FPA2012-35453), DGIID-DGA (grant 2007-E24/2), CPAN (Consolider CSD2007-00042), and by the EU under ITN-STRONGnet (PITN-GA2009-238353). E. Follana is supported on the MICINN Ramón y Cajal program, M. Giordano is supported by the Hungarian Academy of Sciences under "Lendület" grant No. LP2011-011, and A. Vaquero is supported by the Research Promotion Foundation (RPF) of Cyprus under grant $\Pi \mathrm{PO} \Sigma \mathrm{E} \Lambda \mathrm{KY} \Sigma \mathrm{H} / \mathrm{NEO} \Sigma / 0609 / 16$. 


\section{Appendix A. Absence of a sign problem in the infinite-range model}

In this appendix, we prove that the fermion determinant appearing in Eq. (10) is real and positive. To show this, one notices first that $\gamma_{4} \Delta_{x y} \gamma_{4}=\Delta_{x_{P} y_{P}}$, with $x=\left(\vec{x}, x_{4}\right)$ and $x_{P}=\left(-\vec{x}, x_{4}\right)$. Since the determinants of $M$ and $M^{(P)}$ are equal, with $M_{x y}^{(P)}=M_{x_{P} y_{P}}$, one finds that

$$
\begin{aligned}
\operatorname{Det} M=\operatorname{Det} M^{(P)} & =\operatorname{Det}\left[\gamma_{4} \Delta \gamma_{4}+\sigma+i \gamma_{5} \vec{\tau} \cdot \vec{\pi}+i \gamma_{5} \eta+\vec{\tau} \cdot \vec{\rho}\right] \\
& =\operatorname{Det}\left[\Delta+\left(\sigma-i \gamma_{5} \vec{\tau} \cdot \vec{\pi}-i \gamma_{5} \eta+\vec{\tau} \cdot \vec{\rho}\right)\right] .
\end{aligned}
$$

Next, one exploits the unitarity of the matrix $\tilde{C}=\gamma^{1} \gamma^{3}$ and the fact that $\tilde{C} \Delta \tilde{C}^{\dagger}=\Delta^{*}$ to write

$$
\operatorname{Det} M=\operatorname{Det}\left[\Delta^{*}+\left(\sigma-i \gamma_{5} \vec{\tau} \cdot \vec{\pi}-i \gamma_{5} \eta+\vec{\tau} \cdot \vec{\rho}\right)\right] \text {. }
$$

Finally, one performs a rotation $R$ in flavor space in such a way that the vectors $\vec{\pi}_{R}=R \vec{\pi}$ and $\vec{\rho}_{R}=R \vec{\rho}$ lie in the $(1,3)$ plane, i.e., so that $\vec{\rho}_{R} \wedge \vec{\pi}_{R}$ is along direction 2 in flavor space. Since $R$ is implemented by a unitary operator $U_{R}$, and $\tau_{1}$ and $\tau_{3}$ are real, one has that

$$
\begin{aligned}
\operatorname{Det} M & =\operatorname{Det}\left[\Delta^{*}+\left(\sigma-i \gamma_{5} \vec{\tau} \cdot \vec{\pi}_{R}-i \gamma_{5} \eta+\vec{\tau} \cdot \vec{\rho}_{R}\right)\right] \\
& =\operatorname{Det}\left[\Delta+\left(\sigma+i \gamma_{5} \vec{\tau} \cdot \vec{\pi}_{R}+i \gamma_{5} \eta+\vec{\tau} \cdot \vec{\rho}_{R}\right)\right]^{*} \\
& =\operatorname{Det}\left[\Delta+\left(\sigma+i \gamma_{5} \vec{\tau} \cdot \vec{\pi}+i \gamma_{5} \eta+\vec{\tau} \cdot \vec{\rho}\right)\right]^{*}=\operatorname{Det} M^{*} .
\end{aligned}
$$

Finally, since $M$ is diagonal and trivial in color, $M=\bar{M} \mathbf{1}_{C}$, one has that for an even number of colors $\operatorname{det} M=(\operatorname{det} \bar{M})^{N}=\left(\operatorname{det} \bar{M} \operatorname{det} \bar{M}^{\dagger}\right)^{\frac{N}{2}} \geqslant 0$.

\section{Appendix B. Quadratic part of the action in the local model}

In this appendix we report a few results related to the local model discussed in Section 4. We use the following notation:

$$
\begin{aligned}
I_{0}(q, \sigma, z)= & \int_{B} \frac{d^{4} p}{(2 \pi)^{4}} \frac{1}{g\left(p+\frac{q}{2}, z\right) g\left(p-\frac{q}{2}, z\right)}\left\{\sum_{\mu=1}^{4} \sin \left(p_{\mu}+\frac{q_{\mu}}{2}\right) \sin \left(p_{\mu}-\frac{q_{\mu}}{2}\right)\right. \\
& \left.+\left[w_{r}\left(p+\frac{q}{2}\right)+m_{0}+\sigma\right]\left[w_{r}\left(p-\frac{q}{2}\right)+m_{0}+\sigma\right]+z^{2}\right\}, \\
I_{1}(q, \sigma, z)= & \int_{B} \frac{d^{4} p}{(2 \pi)^{4}} \frac{\left[w_{r}\left(p+\frac{q}{2}\right)+m_{0}+\sigma\right]\left[w_{r}\left(p-\frac{q}{2}\right)+m_{0}+\sigma\right]}{g\left(p+\frac{q}{2}, z\right) g\left(p-\frac{q}{2}, z\right)}, \\
I_{2}(q, \sigma, z)= & \int_{B} \frac{d^{4} p}{(2 \pi)^{4}} \frac{1}{g\left(p+\frac{q}{2}, z\right) g\left(p-\frac{q}{2}, z\right)}, \\
I_{3}(q, \sigma, z)= & \int_{B} \frac{d^{4} p}{(2 \pi)^{4}} \frac{w_{r}\left(p+\frac{q}{2}\right)+w_{r}\left(p-\frac{q}{2}\right)+2\left(m_{0}+\sigma\right)}{g\left(p+\frac{q}{2}, z\right) g\left(p-\frac{q}{2}, z\right)} .
\end{aligned}
$$

The non-vanishing entries of the inverse propagator $G_{i j}^{-1}(q)$, Eq. (50), are listed below for the standard Aoki and non-standard Aoki cases. In the unbroken phase the inverse propagator is diagonal, and can be recovered by simply setting $\Pi=0$ or $\eta=0$ in the equations below. Here $\Pi=|\vec{\pi}|$. Standard Aoki phase: 


$$
\begin{aligned}
& G_{\sigma \sigma}^{-1}(q)=8\left\{\frac{\beta_{1}}{4}-I_{0}(q, \sigma, \Pi)+2 I_{1}(q, \sigma, \Pi)\right\}, \\
& G_{\pi_{a} \pi_{b}}^{-1}(q)=8\left\{\left[\frac{\beta_{1}}{4}-I_{0}(q, \sigma, \Pi)\right] \delta_{a b}+2 \pi_{a} \pi_{b} I_{2}(q, \sigma, \Pi)\right\}, \\
& G_{\eta \eta}^{-1}(q)=8\left\{\frac{\beta_{2}}{4}-I_{0}(q, \sigma, \Pi)+2 \Pi^{2} I_{2}(q, \sigma, \Pi)\right\}, \\
& G_{\rho_{a} \rho_{b}}^{-1}(q)=8\left\{\left[\frac{\beta_{2}}{4}-I_{0}(q, \sigma, \Pi)+2 I_{1}(q, \sigma, \Pi)\right] \delta_{a b}+2 I_{2}(q, \sigma, \Pi)\left(\Pi^{2} \delta_{a b}-\pi_{a} \pi_{b}\right)\right\}, \\
& G_{\sigma \pi_{a}}^{-1}(q)=-8 I_{3}(q, \sigma, \Pi) \pi_{a}, \\
& G_{\eta \rho_{a}}^{-1}(q)=-8 I_{3}(q, \sigma, \Pi) \pi_{a} .
\end{aligned}
$$

Non-standard Aoki phase:

$$
\begin{aligned}
& G_{\sigma \sigma}^{-1}(q)=8\left\{\frac{\beta_{1}}{4}-I_{0}(q, \sigma, \eta)+2 I_{1}(q, \sigma, \eta)\right\}, \\
& G_{\pi_{a} \pi_{b}}^{-1}(q)=8\left\{\frac{\beta_{1}}{4}-I_{0}(q, \sigma, \eta)+2 \eta^{2} I_{2}(q, \sigma, \eta)\right\} \delta_{a b}, \\
& G_{\eta \eta}^{-1}(q)=8\left\{\frac{\beta_{2}}{4}-I_{0}(q, \sigma, \eta)+2 \eta^{2} I_{2}(q, \sigma, \eta)\right\}, \\
& G_{\rho_{a} \rho_{b}}^{-1}(q)=8\left\{\frac{\beta_{2}}{4}-I_{0}(q, \sigma, \eta)+2 I_{1}(q, \sigma, \eta)\right\} \delta_{a b}, \\
& G_{\sigma \eta}^{-1}(q)=-8 I_{3}(q, \sigma, \eta) \eta, \\
& G_{\pi_{a} \rho_{b}}^{-1}(q)=-8 I_{3}(q, \sigma, \eta) \eta \delta_{a b} .
\end{aligned}
$$

The first two terms in the small- $q$ expansion of $I_{0}$ and $I_{2}$ is given below.

$$
\begin{aligned}
I_{0}(q, \sigma, z)= & \int_{B} \frac{d^{4} p}{(2 \pi)^{4}} \frac{1}{g(p, z)} \\
& -\frac{q^{2}}{8} \int_{B} \frac{d^{4} p}{(2 \pi)^{4}} \frac{\sum_{\mu=1}^{4}\left(\cos p_{\mu}\right)^{2}+r^{2}\left(\sin p_{\mu}\right)^{2}}{[g(p, z)]^{2}}+\mathcal{O}\left(q^{4}\right) \\
= & I_{0}(0, \sigma, z)-q^{2} I_{0}^{(2)}(\sigma, z)+\mathcal{O}\left(q^{4}\right), \\
I_{2}(q, \sigma, z)= & \int_{B} \frac{d^{4} p}{(2 \pi)^{4}} \frac{1}{[g(p, z)]^{2}} \\
& -\frac{q^{2}}{2} \int_{B} \frac{d^{4} p}{(2 \pi)^{4}} \frac{\sum_{\mu=1}^{4}\left(\sin p_{\mu}\right)^{2}\left[\cos p_{\mu}+r\left(w_{r}(p)+m_{0}+\sigma\right)\right]^{2}}{[g(p, z)]^{4}}+\mathcal{O}\left(q^{4}\right) \\
= & I_{2}(0, \sigma, z)-q^{2} I_{2}^{(2)}(\sigma, z)+\mathcal{O}\left(q^{4}\right) .
\end{aligned}
$$

Notice that

$$
I_{0}(0, \sigma, z)=\int_{B} \frac{d^{4} p}{(2 \pi)^{4}} \frac{1}{g(p, z)}= \begin{cases}\frac{\beta_{1}}{4}, & \text { in the standard Aoki phase } \\ \frac{\beta_{2}}{4}, & \text { in the non-standard Aoki phase. }\end{cases}
$$




\section{References}

[1] V. Azcoiti, A. Nakamura, Phys. Rev. D 27 (1983) 2559.

[2] H.W. Hamber, Nucl. Phys. B 251 (1985) 182.

[3] S. Aoki, Phys. Rev. D 30 (1984) 2653.

[4] S. Aoki, Phys. Rev. Lett. 57 (1986) 3136.

[5] S. Aoki, S. Boettcher, A. Gocksch, Phys. Lett. B 331 (1994) 157.

[6] M. Creutz, arXiv:hep-lat/9608024.

[7] K.M. Bitar, Phys. Rev. D 56 (1997) 2736.

[8] S. Aoki, T. Kaneda, A. Ukawa, Phys. Rev. D 56 (1997) 1808.

[9] K.M. Bitar, U.M. Heller, R. Narayanan, Phys. Lett. B 418 (1998) 167.

[10] R.G. Edwards, U.M. Heller, R. Narayanan, R.L. Singleton Jr., Nucl. Phys. B 518 (1998) 319.

[11] S.R. Sharpe, R.L. Singleton Jr., Phys. Rev. D 58 (1998) 074501.

[12] S. Aoki, Nucl. Phys. B (Proc. Suppl.) 60A (1998) 206.

[13] K. Bitar, Nucl. Phys. B (Proc. Suppl.) 63A-C (1998) 829.

[14] S. Sharpe, R.L. Singleton Jr., Nucl. Phys. B (Proc. Suppl.) 73 (1999) 234.

[15] R. Kenna, C. Pinto, J.C. Sexton, Phys. Lett. B 505 (2001) 125.

[16] M. Golterman, Y. Shamir, Phys. Rev. D 68 (2003) 074501.

[17] E.M. Ilgenfritz, W. Kerler, M. Müller-Preussker, A. Sternbeck, H. Stuben, Phys. Rev. D 69 (2004) 074511.

[18] A. Sternbeck, E.M. Ilgenfritz, W. Kerler, M. Müller-Preussker, H. Stuben, Nucl. Phys. B (Proc. Suppl.) 129-130 (2004) 898.

[19] M. Golterman, S.R. Sharpe, R.L. Singleton Jr., Nucl. Phys. B (Proc. Suppl.) 140 (2005) 335.

[20] E.M. Ilgenfritz, M. Müller-Preussker, M. Petschlies, K. Jansen, M.P. Lombardo, O. Philipsen, L. Zeidlewicz, A. Sternbeck, PoS Lattice2007 (2008) 238.

[21] V. Azcoiti, G. Di Carlo, A. Vaquero, Phys. Rev. D 79 (2009) 014509.

[22] S. Sharpe, Phys. Rev. D 79 (2009) 054503.

[23] V. Azcoiti, G. Di Carlo, E. Follana, A. Vaquero, PoS LATTICE2009 (2009) 068.

[24] V. Azcoiti, G. Di Carlo, E. Follana, A. Vaquero, PoS LATTICE2010 (2010) 091.

[25] V. Azcoiti, G. Di Carlo, E. Follana, A. Vaquero, PoS LATTICE2011 (2011) 112.

[26] G. Akemann, P.H. Damgaard, K. Splittorff, J.J.M. Verbaarschot, Phys. Rev. D 83 (2011) 085014.

[27] M. Kieburg, K. Splittorff, J.J.M. Verbaarschot, Phys. Rev. D 85 (2012) 094011.

[28] C. Vafa, E. Witten, Phys. Rev. Lett. 53 (1984) 535.

[29] V. Azcoiti, A. Galante, Phys. Rev. Lett. 83 (1999) 1518.

[30] X. Ji, Phys. Lett. B 554 (2003) 33.

[31] P.R. Crompton, Phys. Rev. D 72 (2005) 076003.

[32] V. Azcoiti, G. Di Carlo, E. Follana, A. Vaquero, JHEP 1007 (2010) 047.

[33] K.M. Bitar, P.M. Vranas, Phys. Rev. D 50 (1994) 3406.

[34] V. Azcoiti, G. Di Carlo, E. Follana, A. Vaquero, Nucl. Phys. B 870 (2013) 138.

[35] A. Dhar, R. Shankar, S.R. Wadia, Phys. Rev. D 31 (1985) 3256.

[36] D. Ebert, M. Nagy, M.K. Volkov, Phys. Atom. Nucl. 59 (1996) 140;

D. Ebert, M. Nagy, M.K. Volkov, Yad. Fiz. 59 (1996) 149.

[37] D. Ebert, H. Reinhardt, M.K. Volkov, Prog. Part. Nucl. Phys. 33 (1994) 1. 